\title{
Natural Images: A Lingua Franca for Primates?
}

\author{
Natasha Sigala* \\ Department of Experimental Psychology, University of Oxford and MRC - Cognition and Brain Sciences Unit, \\ Cambridge, $U K$
}

\begin{abstract}
Primates in the laboratory often perform tasks that involve discrimination of pictures shown on computer screens. It is not clear, however, if they perceive the pictures as symbolic representations of real objects. In this study we tested the ability of two monkeys (Macaca mulatta) to categorize pictures on a computer screen. The pictures were photographs of objects from 17 categories, which were grouped as natural and familiar, natural and unfamiliar, or artificial and unfamiliar. The monkeys learnt the categories of the natural and familiar objects faster than those of the artificial and unfamiliar objects. Nevertheless, once they had learnt the categories, they were just as fast and accurate at generalizing to new exemplars of artificial and unfamiliar objects. This finding suggests that the monkeys are able to extract similarities and form categories from a small number of stimuli differing widely across size, color and viewpoint, and for which they have no prior internal representation.
\end{abstract}

Keywords: Rhesus monkey, categorization, learning.

\section{INTRODUCTION}

It has been reported that members of remote cultures, who are not familiar with pictorial material, do not recognize pictures of familiar faces and objects effortlessly [1]. In addition, familiarity with pictures, as a means of symbolic representation, can be more important for recognition than familiarity with the depicted objects [2]. On the other hand, pigeons and other animals that get extensive practice in the laboratory are able to perform a variety of visual discriminations with pictures, e.g. [3-6]. In particular, research in primate recognition often employs monkeys performing tasks with pictures shown on computer screens, e.g. [7-10]. However, it is not clear if the monkeys perceive the pictures as symbolic representations of familiar objects. We trained two macaque monkeys (Macaca mulatta) on a categorization task with natural images from 17 categories, ranging from familiar and natural to unfamiliar and artificial. We hypothesized that if the monkeys perceive the pictures they see on the monitor as representations of familiar objects, then they should learn categories of familiar, natural stimuli faster than categories of unfamiliar, man-made stimuli. Here we present evidence that familiarity of the monkeys with the depicted objects can facilitate learning of their pictorial representations. We also report that in the generalization phase, they are just as fast and accurate at categorizing new examples of artificial as well as familiar objects. This finding suggests that the monkeys are able to extract similarities and form equivalence classes from a small number of stimuli differing widely across size, color and viewpoint, and for which they have no prior internal representation.

*Address correspondence to this author at the MRC Cognition and Brain Sciences Unit, 15 Chaucer Road, CB2 7EF Cambridge, UK;

E-mail: natasha.sigala@mrc-cbu.cam.ac.uk

\section{MATERIALS AND METHODS}

We trained two adult, male rhesus monkeys (Macaca mulatta), weighing 11.0 and $13.0 \mathrm{~kg}$., to perform a categorization task with natural images following standard operant conditioning techniques. They had been trained on other discrimination tasks with colored shapes, but had no experience with pictures. The images $(n=210)$ were presented on a computer screen and represented natural, familiar (humans, monkeys, food items), natural but not familiar (e.g. bears, snakes), or man-made, not familiar objects (ships, buses, airplanes) (total of 17 categories) (Fig. 1). All studies were in compliance with the guidelines of the European Community (EUVD 86/609/EEC) for the care and use of laboratory animals.

Each color image was shown at 256x256 pixels resolution, subtended $5^{0} \times 5^{0}$ visual angle, and were presented on a 21 " monitor. The timing of stimulus presentation, the responses and juice reward were computer controlled.

The animals were seated in a primate chair with two response levers mounted at the front. During the learning phase they were familiarized with a subset of images (4 per category) and learned by trial-and-error to respond by pulling one of the levers. The monkeys were familiarized with images sorted by natural category and trained to respond to each one of them by pulling one of two levers. The lever assignment was predetermined by the experimenter. The images represented natural, familiar, natural but not familiar, or artificial, not familiar objects. The left lever categories were: bears, wild cats, monkeys, chimpanzees, parrots, food items, ships and airplanes. The right lever categories were: snakes, salamanders, humans and portraits of human faces, gorillas, orangutans, flowers, leaves and buses. The mean number of exemplars in each category was twelve. We considered as natural and familiar the categories of monkeys, humans and food items. We considered as artificial and not familiar the categories of airplanes, buses, ships and por- 
A
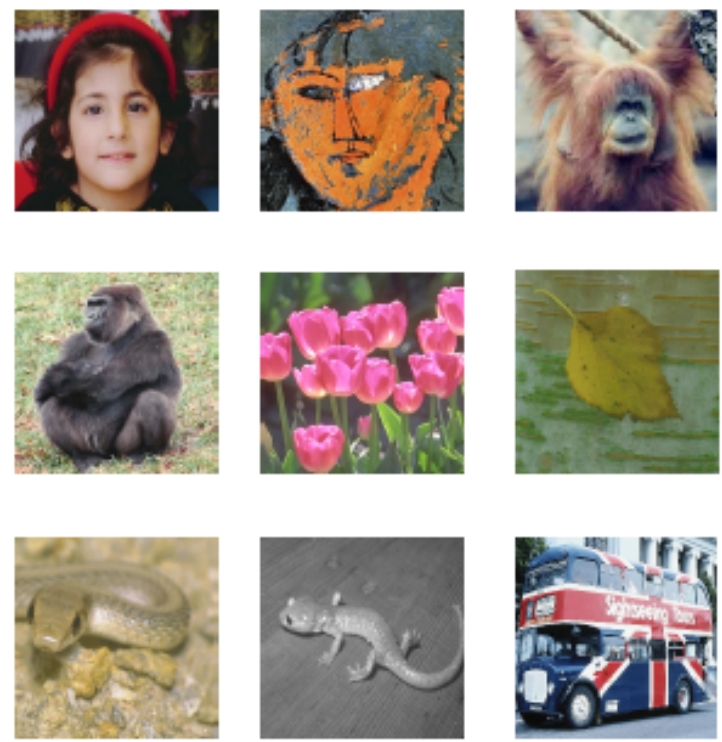

\section{Left lever categories}
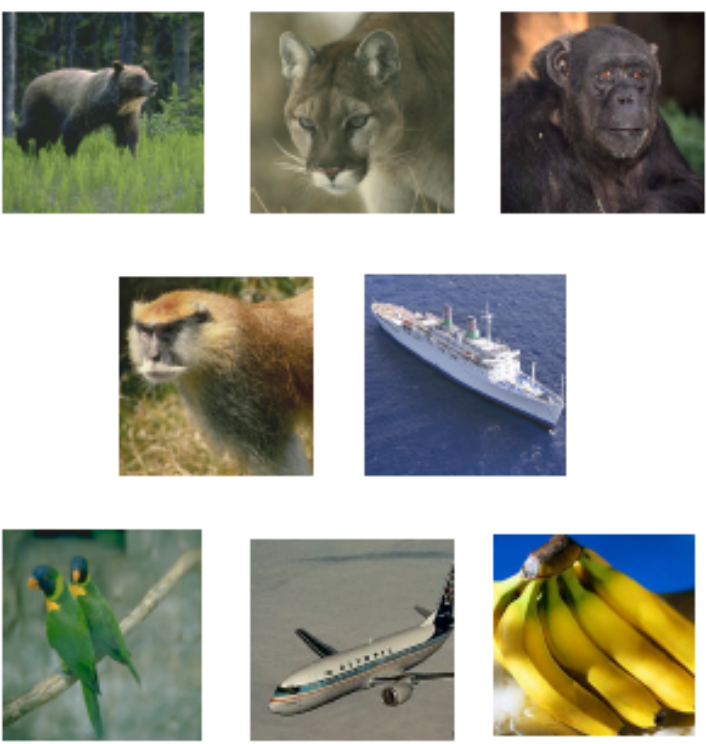

B
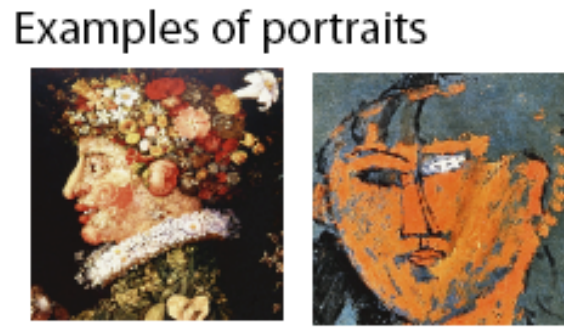

\section{Examples of bears}
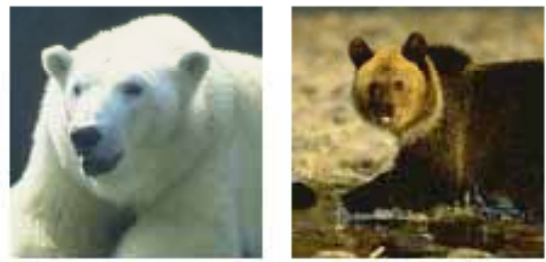

\section{Examples of food items}
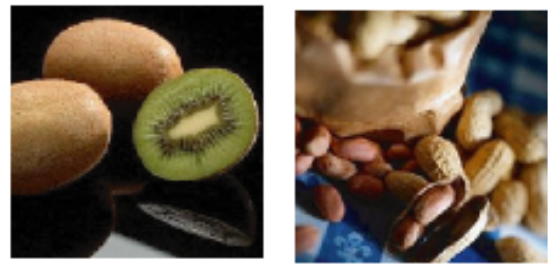

\section{Examples of airplanes}
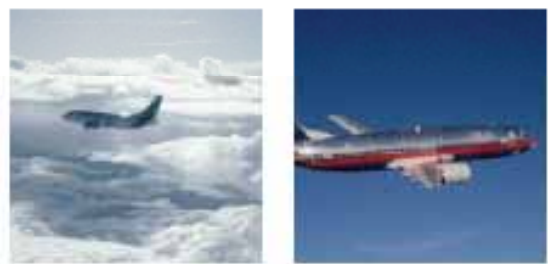

Fig. (1). A) Examples of the images presented in the corresponding categories. B) Examples of the within category variability.

traits. The rest of the categories were considered as natural, but not familiar to monkeys that were raised in captivity. During both learning and generalization phase blocks of trials of a particular category were intermixed with blocks of trials from other categories, and never in isolation. The generalization phase started after learning of all categories had been completed. At the beginning of each trial a fixation spot $\left(1^{0} \times 1^{0}\right)$ appeared in the middle of the screen. After $500 \mathrm{~ms}$ the fixation spot was switched off and a stimulus was presented until the monkey pulled a lever or for up to $2500 \mathrm{~ms}$. The monkeys received auditory feedback for correct responses (high-pitched tone) and were rewarded with a drop of juice. For incorrect responses a delay of 2 seconds was imposed.

We used a criterion of four consecutive correct categorizations for a particular image as a measure of learning, which indicated stable correct performance (chance level
6.25\%). If "i" stands for "incorrect" and "c" for "correct", then for the hypothetical response sequence "cicicicccc", the learning criterion is achieved after ten trials. During the learning phase, the animals were exposed to four exemplars per category. If the learning criterion is achieved in 9, 28, 9 and 8 trials for each image respectively, then the category is acquired within nine trials (median).

\section{RESULTS}

Table 1 reports the median number of trials to the learning criterion for the first 4 stimuli that were used to define a category (learning phase). The monkeys learned all categories with a minimum of 4 and a maximum of 23 (median value) trials (see Materials and Methods for learning criterion). To compare the performance of the 2 animals in the different types of categories, we performed a 2 (subject: M1 
and M2) x 3 (type of category: natural and familiar, natural and unfamiliar, artificial and unfamiliar) ANOVA on the mean number of trials it took them to acquire the learning criterion. Only the main effect of type of category was significant $\left(\mathrm{F}_{1,3}=5,97, \mathrm{p}=0.006\right)$, indicating that both animals learned more easily the categories that depicted familiar objects.

Table 1. Order in which the Categories were Introduced to the Monkeys

\begin{tabular}{|c|c|}
\hline M1 & M2 \\
\hline \hline Flowers (6) & Flowers (5) \\
\hline Bears (9) L & Bears (5) L \\
\hline Leaves (6) & Wild Cats (10) L \\
\hline Parrots (4) L & Leaves (7) \\
\hline Snakes (9) & Parrots (9) L \\
\hline Monkeys (4) L & Monkeys (4) L \\
\hline Humans (6) & Snakes (4) \\
\hline Salamanders (4) & Salamanders (9) \\
\hline Chimpanzees (9) L & Chimpanzees (4) L \\
\hline Portraits (17) & Humans (4) \\
\hline Ships (23) L & Portraits (8) \\
\hline Buses (12) & Ships (11) L \\
\hline Wild Cats (4) L & Buses (10) \\
\hline Orangutans (4) & Gorillas (5) \\
\hline Gorillas (6) & Orangutans (7) \\
\hline Airplanes (12) L & Food items (14) L \\
\hline Food Items (10) L & Airplanes (9) L \\
\hline
\end{tabular}

In brackets: number of trials to learning criterion (see Materials and Methods).

$\mathrm{L}$ : category assigned to the left lever.

After the monkeys had reached the learning criterion for the first 4 stimuli that were used to define a category, they were shown a total of 8 new images to categorize in the classes they had learned. A two-way ANOVA contrasting the mean reaction times (RTs) to the first presentation of the first 4 category exemplars (learning phase) with the RTs to the first presentations of the last 4 category exemplars (generalization phase) showed a significant main effect for the two phases (learning vs. generalization) $\left(\mathrm{F}_{1,2}=6,77, \mathrm{p}=0.014\right)$ but no effect of category type $\left(\mathrm{F}_{1,3}=0.05, \mathrm{p}=0.95\right)$. During the generalization phase the new images were presented randomly interleaved and not in blocks of the same category. The mean RT during learning of first 4 exemplars was $987+/-62 \mathrm{~ms}$ (SEM, standard error of the mean), while the mean RT for the last 4 exemplars was $779+/-50 \mathrm{~ms}$ for all category types. A further comparison of the RTs and correct responses to natural and artificial stimuli with a nonparametric test showed no differences across the different categories (two-tailed Mann-Whitney, $\mathrm{a}=0.001$ ). Hence, in the generalization phase and after little training, the monkeys were able to categorize correctly new images, including those of artificial objects.

\section{DISCUSSION}

The monkeys learned all the categories within the first session they were introduced. There may be several reasons for that. The first might be the fact that, in contrast to previous relevant studies [11], the monkeys did not have to discriminate stimuli on the basis of the presence or absence of the positive stimulus (compare learning rates in experiments 1 and 2 in [11] and also see [12]). Moreover, they were not presented with the same category as positive and then as a negative category [6]. All pictures were assigned to a category and the lever assignment remained the same throughout training and testing. A second reason for the fast learning rates may be the quality of the stimuli. The pictures consisted of relatively close, full figures of the category objects (Fig. 1) and can be characterized as good stimuli (as opposed to poor) [11] based on the size and the position of the main object in the picture.

There are similarities and differences in the data from the two animals. For both of them, the categories that were learned more easily ( 4 trials to learning criterion) were categories of animals. For M1, 2 of the 5 and for M2, 3 of the 4 easiest categories were primates: monkeys and orangutans,

Table 2. Median Number of Trials to Learning Criterion

\begin{tabular}{|c|c|c|c|}
\hline \multicolumn{2}{|c|}{ M1 } & \multicolumn{2}{|c|}{ M2 } \\
\hline Category & \# Trials & Category & \# Trials \\
\hline Monkeys & 4 & Chimpanzees & 4 \\
\hline Orangutans & 4 & Monkeys & 4 \\
\hline Parrots & 4 & Humans & 4 \\
\hline Salamanders & 4 & Snakes & 4 \\
\hline \multirow[t]{2}{*}{ Wild Cats } & 4 & & \\
\hline & & Bears & 5 \\
\hline Flowers & 6 & Flowers & 5 \\
\hline Gorillas & 6 & Gorillas & 5 \\
\hline Humans & 6 & & \\
\hline \multirow[t]{2}{*}{ Leaves } & 6 & Leaves & 7 \\
\hline & & Orangutans & 7 \\
\hline Bears & 9 & & \\
\hline Chimpanzees & 9 & Portraits & 8 \\
\hline \multirow[t]{2}{*}{ Snakes } & 9 & & \\
\hline & & Airplanes & 9 \\
\hline \multirow[t]{2}{*}{ Food items } & 10 & Parrots & 9 \\
\hline & & Salamanders & 9 \\
\hline Airplanes & 12 & & \\
\hline \multirow[t]{2}{*}{ Buses } & 12 & Buses & 10 \\
\hline & & Wild Cats & 10 \\
\hline \multirow[t]{2}{*}{ Portraits } & 17 & & \\
\hline & & Ships & 11 \\
\hline \multirow[t]{2}{*}{ Ships } & 23 & & \\
\hline & & Food items & 14 \\
\hline
\end{tabular}


and chimpanzees, monkeys and humans, respectively (Table 2). All natural categories were learned with a minimum of 4 and a maximum of 14 trials (median value), whereas all categories of man-made objects were learned with a minimum of 8 (portraits for M2) and with a maximum of 23 trials. A surprising similarity for both animals was the difficulty to learn the category of food items that during the learning phase consisted of fruit only (10 trials for M1, 14 trials for M2). A possible reason for this might be the fact that fruit images often included leaves, another source of food for the monkeys, which consisted a separate category mapped on the opposite lever. A second possible explanation is that, in contrast to the rest of the categories, food items was a superordinate category, including a variety of basic fruit types (e.g. bananas, apples). There is evidence that monkeys encode this category by type of fruit (i.e. at the basic level) [13], therefore it may have been more difficult for them to form the more abstract, superordinate fruit category. The fact that it was one of the last categories that were introduced to the monkeys might also have played a role, although the categories that were presented immediately before them were learned faster (gorillas for M1 with 6 trials, and orangutans for M2 with 7 trials). An interesting difference between the two monkeys was that they showed a somewhat individual preference or difficulty while learning the different categories. For example, the category Wild Cats was one of the 5 easiest for M1, but one of the 4 most difficult for M2. This finding supports the idea that the ability to associate pictures and objects may not be uniformly distributed within a group of subjects and may depend on individual factors $[10,14,15]$, cf. [16] for differences in categorization of conspecific pictures between the two animals.

The effect of familiarity that was observed during the learning phase could be interpreted as evidence that the monkeys perceive the stimuli presented on the computer screen as representations of objects they encounter in their everyday life. This result is in agreement with other studies that report successful categorizations of natural categories, like animals and trees, e.g. [7, 8], and recognition of photographic representations of objects with experience [17]. Moreover, the observation that as few as 4 pictures suffice for the monkeys to form an adequate category representation of objects they have never experienced in their life is novel. This also seems to be the case for objects that the monkeys (or any other animal) are not expected to be genetically disposed to recognize (i.e. ships, buses, airplanes). This result is in agreement with the reported ability of untrained infant monkeys to categorize scale models of animals and furniture [18]. Both animals were just as fast and accurate at generalizing to new examples of man-made objects, suggesting that they are able to extract similarities and form equivalence classes, from a small number of stimuli differing widely across size, color and viewpoint, and for which they have no prior internal representation.

\section{ACKNOWLEDGEMETNS}

This work was carried out at the laboratory of Prof. N.K. Logothetis, MPI for Biological Cybernetics, Tuebingen, Germany and funded by the Max Planck Society. NS is supported by the Royal Society (DH051644).

\section{REFERENCES}

[1] Deregowski JB, Muldrow ES, Muldrow WF. Pictorial recognition in a remote Ethiopian population. Perception 1972; 1(4): 417-25.

[2] Deregowski J. Pictorial perception and culture. Sci Am 1972; 227: 82-8.

[3] Hernstein RJ, Loveland DH, Cable C. Natural concepts in pigeons. J Exp Psychol: Anim Behav Process 1976; 2(4): 285-302.

[4] Cerella J. Visual classes and natural categories in the pigeon. J Exp Psychol: Hum Percept Perform 1979; 5(1): 68-71.

[5] Watanabe S, Ito Y. Discrimination of individuals in pigeons. Bird Behav 1991; 9(1-2): 20-9.

[6] Roberts WA, Mazmanian DS, Concept learning at different levels of abstraction by pigeons, monkeys, and people. J Exp Psychol: Anim Behav Process 1988; 14(3): 247-60.

[7] Fabre-Thorpe M, Richard G, Thorpe SJ. Rapid categorization of natural images by rhesus monkeys. Neuroreport 1998; 9(2): 303-8.

[8] Vogels R. Categorization of complex visual images by rhesus monkeys. Part I: behavioural study. Eur J Neurosci 1999; 11(4): 1223-38

[9] Sheinberg DL, Logothetis NK. Noticing familiar objects in real world scenes: the role of temporal cortical neurons in natural vision. J Neurosci 2001; 21: 1340-50.

[10] Hampson RE, Pons TP, Stanford TR, Deadwyler SA. Categorization in the monkey hippocampus: a possible mechanism for encoding information into memory. Proc Natl Acad Sci USA 2004; 101(9): 3184-9.

[11] Schrier AM, Angarella R, Povar ML. Studies of concept formation by stumptailed monkeys: concepts humans, monkeys, and letter A. J Exp Psychol: Anim Behav Process 1984; 10(4): 564-84.

[12] Schrier AM, Brady PM. Categorization of natural stimuli by monkeys (Macaca mulatta): effects of stimulus set size and modification of exemplars. J Exp Psychol: Anim Behav Process 1987; 13(2): 136-43.

[13] Sands SF, Lincoln CE, Wright AA. Pictorial similarity judgments and the organization of visual memory in the rhesus monkey. $\mathrm{J}$ Exp Psychol: Gen 1982; 111(4): 369-89.

[14] Fagot J, Ed. Picture perception in animals. Hove: Psychology Press 2000.

[15] Martin-Malivel J, Fagot J. Cross-modal integration and conceptual categorization in baboons. Behav Brain Res 2001; 122(2): 209-13.

[16] Koba R, Izumi A. Sex categorization of conspecific pictures in Japanese monkeys (Macaca fuscata). Anim Cogn 2006; 9(3): 18391.

[17] Buckley MJ, Gaffan D. Learning and transfer of object-reward associations and the role of the perirhinal cortex. Behav Neurosci 1998; 112(1): 15-23.

[18] Murai C, Tomonaga M, Kamegai K, Terazawa N, Yamaguchi M. Do infant Japanese macaques (Macaca fuscata) categorize objects without specific training? Primates 2004; 45(1): 1-6.

(C) Natasha Sigala; Licensee Bentham Open.

This is an open access article licensed under the terms of the Creative Commons Attribution Non-Commercial License (http://creativecommons.org/licenses/by-nc/3.0/) which permits unrestricted, non-commercial use, distribution and reproduction in any medium, provided the work is properly cited. 